\title{
A COMPARISON OF DIFFERENT TECHNOLOGIES FOR THRUST VECTORING IN A LINEAR AEROSPIKE
}

\section{MICHELE FERLAUTO ${ }^{1}$, ANDREA FERRERO ${ }^{2}$, MATTEO MARSICOVETERE $^{3}$ AND ROBERTO MARSILIO ${ }^{4}$}

\author{
${ }^{1}$ Politecnico di Torino \\ Corso Duca degli Abruzzi 24, 10129 Torino, Italy \\ michele.ferlauto@polito.it \\ 2 Politecnico di Torino \\ Corso Duca degli Abruzzi 24, 10129 Torino, Italy \\ andrea_ferrero@polito.it \\ ${ }^{3}$ Politecnico di Torino \\ Corso Duca degli Abruzzi 24, 10129 Torino, Italy \\ matteo.marsicovetere@studenti.polito.it \\ ${ }^{4}$ Politecnico di Torino \\ Corso Duca degli Abruzzi 24, 10129 Torino, Italy \\ roberto.marsilio@polito.it
}

Key words: Aerospike; Shock Vectoring; Differential Throttling

\begin{abstract}
The aerospike nozzle represents an interesting technology for Single-Stage-To-Orbit vehicles because of its self-adapting capability. It is possible to get thrust vectoring capabilities in different ways. A straightforward solution consists in applying differential throttling to multiple combustion chambers which feed the nozzle. An alternative technology, which can be used in the presence of a common combustion chamber, is represented by fluidic thrust vectoring which requires the injection of a secondary flow from a slot on the wall. In this work, the flow field in a linear aerospike nozzle is numerically investigated by means of RANS simulations and both differential throttling and shock vectoring are studied. A parametric study is performed to evaluate the potential of the two technologies.
\end{abstract}

\section{Introduction}

Rocket engines used in space launchers are usually equipped with conventional fixed geometry bell shaped nozzle which provide good performances and are quite reliable. However, there are launchers configurations in which there is a core engine which has to work from sea-level to almost vacuum conditions, like the Vulcain 2 engine in the Ariane 5 launcher or the Space Shuttle Main Engines in the Space Shuttle launching system. Furthermore, Single-Stage-to-Orbit (SSTO) configurations have been also proposed. In all these applications a significant performance gain could be obtained by reducing the non-adaptation losses [10]. 
The aerospike nozzle represents an effective self-adapting nozzle which allows to adapt the nozzle exit pressure according to the environment pressure without the use of any moving component. Several configurations of aerospike engines have been proposed and investigated, with both circular and linear designs $[13,4,16,22,21,11]$. In the 1960s, Rocketdyne developed the J-2T-200K and J-2T-250K annular engines which were based on a toroidal combustion chamber and a truncated plug [19]. These engines, which were derived from the J-2 engine used in the Saturn-family launchers, were evaluated with both cold and hot tests.

The linear aerospike engine XRS-2200 was selected as a candidate propulsion system for the Venture Star/X33 SSTO spaceplane in the 1990s [3].

After these pioneering contributions, the research effort on aerospikes slowed down because of the cancellation of several programs. More recently, the research in field has been lead by universities and private companies $[2,1]$.

The choice of the aerospike technology requires particular attention when dealing with thrust vectoring. For example, it can be difficult to use a gimballed joint because of the large diameter/extension of aerospike nozzle which prevents the possibility of moving the entire nozzle. However, thrust vectoring is a key requirement for the use of aerospike nozzle in real applications. For these reasons, several alternative thrust vectoring technologies have been investigated [1].

A straightforward control strategy is represented by differential throttling which can be applied in the presence of clustered aerospike engines with multiple independent combustion chamber. Since the mass flow rate and the pressute

lateral thrust component wi However, there are both chamber is considered. represented by fluidic thru order to create an obstacle for the primary flow con
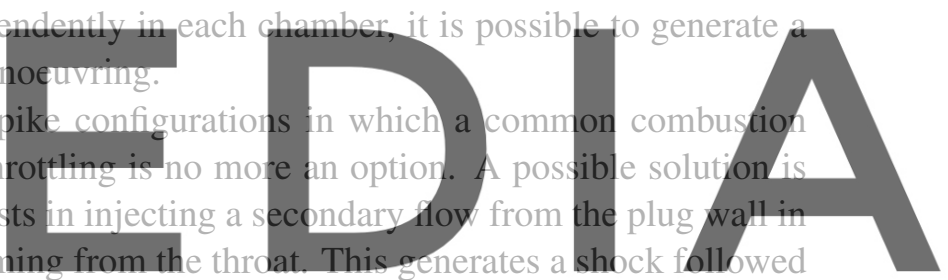

by a separation downstream to the injection point.

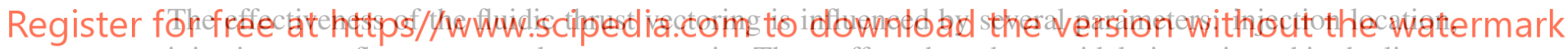
injection mass flow rate, nozzle pressure ratio. These effects have been widely investigated in the literature with both experimental $[5,6,7]$ and numerical studies $[5,6,15,9,18,14]$.

In the present work, differential throttling and fluidic thrust vectoring are investigated and compared as candidate technologies for thrust control in a linear aerospike. The performances of the control strategies are evaluated in terms of lateral force component magnitude by means of numerical simulations.

\section{THRUST IN AN AEROSPIKE NOZZLE}

Consider the generic rocket nozzle represented in Figure 1a. The area of the exit section of the nozzle is $A_{e}$. The thrust $F$ generated by the rocket can be computed as:

$$
F=\left(F_{x}, F_{y}\right)^{T}=\int_{A_{e}}\left(\rho(u \cdot n) u+\left(p-p_{0}\right) n\right) d A
$$

where $\rho, u, p, p_{0}$ and $n$ represent density, velocity vector, pressure, ambient pressure and normal unit vector, respectively. According to the scheme in Figure 1a, the axis of the nozzle is assumed inclined 


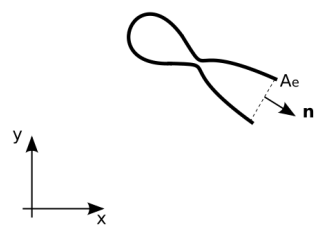

(a)

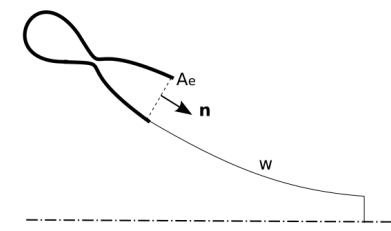

(b)

Figure 1: Scheme for the computation of the thrust component in a inclined bell nozzle (a) and in an aerospike represented by an inclined bell nozzle followed by a plug (b).

with respect to the global reference system $(x, y)$.

Consider now a linear aerospike nozzle as represented in Fig. 1b: the Figure shows only the upper half because of symmetry considerations. The previous expression can be updated in order to compute the force in the new configuration:

$$
F=\int_{A_{e}}\left(\rho(u \cdot n) u+\left(p-p_{0}\right) n\right) d A+\int_{w}\left(\left(p-p_{0}\right) \mathbb{I}+\tau\right) \cdot n d A
$$

where II represents the identity matrix and $\tau$ is the viscous stress tensor at wall. The first integral in Eq. 2 represents the thrust of a rocket with a nozzle truncated at the section $A_{e}$. This term is augmented by the force that the fluid applies to the plug which contains both pressure and viscous contributions. Since the aerospike is symmetrie the in tegrals which appear in Eq. 2 must be extended to both the r and to the full plug surface.

The terms in Eq.2 put in nozzle to produce a latera
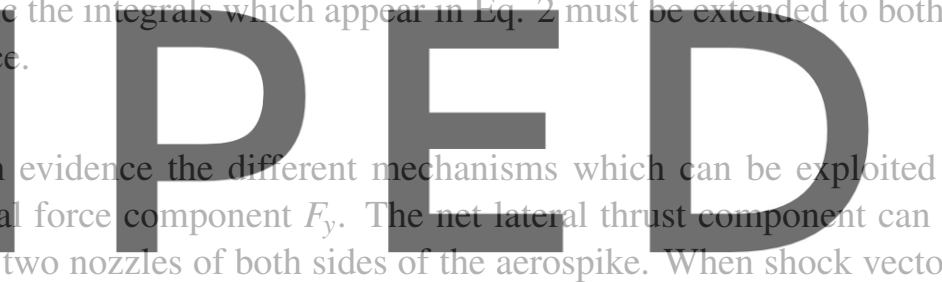

applying the Eq. 2 to the two nozzles of both sides of the aerospike. When shock vectoring is applied a

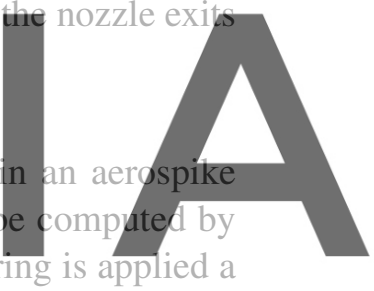

fluid is injected on one side of the plug: this leads to an asymmetric pressure distribution on the two sides

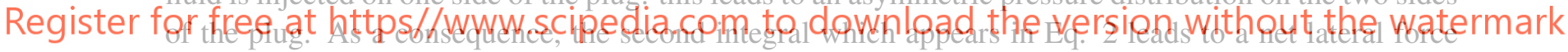

component. However, the first integral in Eq. 2 does not give any contribution in this thrust vectoring

strategy because the perturbations induced by the injected fiuid influence only the plug region and do not affect the flow field upstream to the section $A_{e}$.

An alternative effect is exploited when differential throttling is applied. In this case a different total pressure is imposed in the combustion chambers which feed the two sides of the plug. As a consequence both the integrals which appear in Eq. 2 contributes to the generation of the lateral force components: the first integral takes into account the force contributions generated by the region upstream to the section $A_{e}$ while the second integral quantifies the pressure unbalance on the two sides of the plug.

\section{NUMERICAL SIMULATION SETUP}

A preliminary validation of the model and a grid convergence analysis was performed [8] on the results related to a cold flow experimental test [10]. In the present simulations the fluid is assumed to be air with frozen chemical composition. The flow field is described by means of the compressible Reynoldsaveraged Navier-Stokes (RANS) equations with the Spalart-Allmaras closure model [20]. An ideal gas which follows the ideal gas equation of state is assumed. The specific heat ratio is assumed constant and 


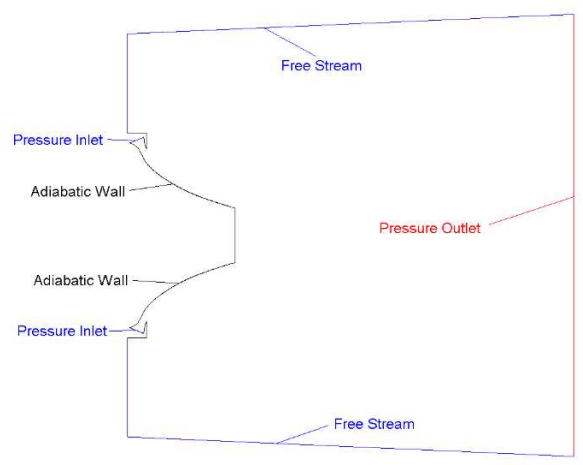

Figure 2: Computational domain.

set to $\gamma=1.4$. The dynamic viscosity is evaluated by means of the Sutherland's law. All the simulations reported in the following were performed by assuming a nozzle pressure ratio (chamber total pressure over ambient static pressure) equal to 56.7

The governing equations are solved by means of a second order accurate finite volume discretisation implemented in the Fluent solver. Convective fluxes are evaluated by means of the Roe approximate Riemann solver [17]. The computational domain is reported in Figure 2 which shows the boundary conditions. In particular, total temperature, total pressure and angle are imposed at the nozzle inlet. The mesh contains approximately 110000 cells.

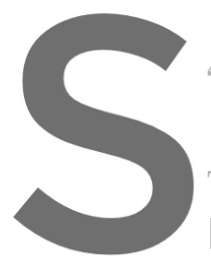

4 COMPARISON OF DIFFERENTIAL THROTTLING ANI I ING

The simulations are performed [12].
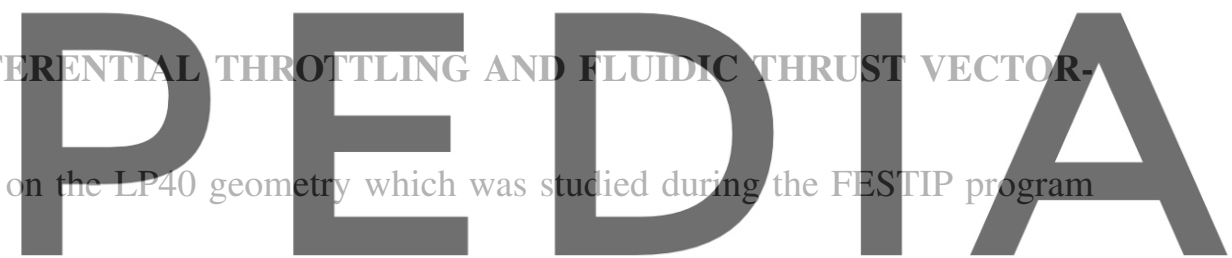

Figure 3 shows the Mach field obtained on the LP40 plug by different control strategy. In Figure 3a

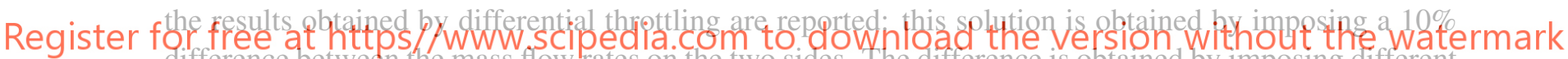
difference between the mass flow rates on the two sides. The difference is obtained by imposing different

total pressure with the same total temperature.

In Figure $3 \mathrm{~b}$ the flow field obtained by shock vector control is reported. It is possible to clearly identify the location of the secondary injection on the upper side of the plug: the secondary flow acts as an obstacle which induces a shock wave an a separation. The separation moves upstream with respect to the injection slot and generates a sort of fluidic ramp on the wall. These results refer to a ratio between the secondary and the primary mass flow rate equal to $10 \%$ and the injection slot is located at $60 \%$ of the plug lenth.

The results in Figure 3c are qualitatively similar to what observed in Figure $3 \mathrm{~b}$ but now the injection slot is located at $90 \%$ of the plug length. The consequences induced by the choice of the injection location can be seen more clearly by comparing the wall pressure distribution which is reported in Figure 4 for both injection locations. The results show that the secondary injection generates a shock wave in front of the injection slot. The shock wave is not exactly located on the injection slot but is placed upstream because of the boundary layer separation. fact is that an expansion region is present downstream to the injection slot. The magnitude of the lateral force component is determined by the integral of the wall pressure distribution on the full plug: the compression and the expansion regions lead to opposite contri- 
butions which tend to cancel each other. This effect is particularly evident when the injection is located at $60 \%$ of the plug length (see Figure $4 a$ ). In contrast, when the injection is performed at $90 \%$ of the plug length, the surface interested by the expansion is reduced, as shown by Figure $4 \mathrm{~b}$.

These qualitative considerations are confirmed by the quantitative force evaluations reported in the plot of Figure 5a. In particular, the lateral thrust component normalised with respect to the magnitude of the unperturbed thrust is shown as a function of the secondary mass flow rate percentage. When the injection is performed at $90 \%$ of the plug length the behaviour is monotone and the lateral thrust component is always higher with respect to the results obtained when the injection is performed at $60 \%$ of the plug length. This seems to be related to the fact that, when the injection is performed close to the end, the expansion acts on a small region and is not able to significantly reduced the effects generated by the compression. These results are in line with the experimental finding of [5].

Furthermore, when the injection is performed at $60 \%$ of the plug length the results are non-monotone with respect to the secondary mass flow rate percentage: this could be again related to the absence of a clear net effeet since both compression and expansion can influence a significant portion of the plug.

Finally, the lateral thrust component obtained by differential throttling is reported in Figure 5b: the trend in this case is very clear. The lateral thrust component grows linearly with the difference between the mass flow rates in the nozzles on the two sides of the plug.

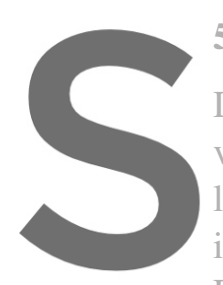

5 CONCLUSIONS

Differential throttling and shock

vectoring in an aerospik

lateral thrust componen

in the chambers which
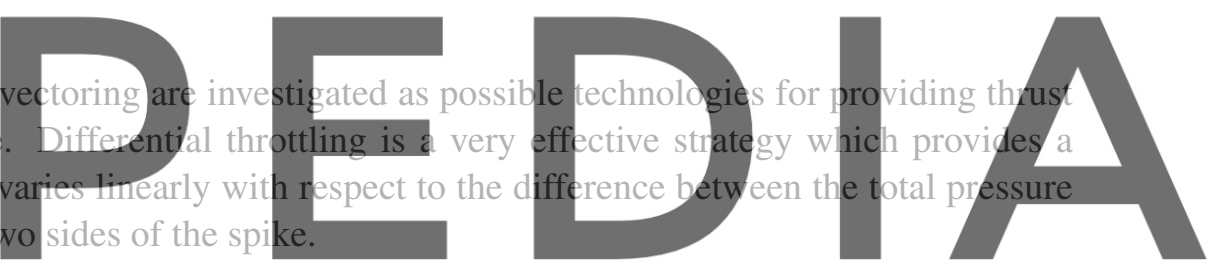

Fluidic thrust vectoring and in particular shock vectoring represents a valid alternative which could be

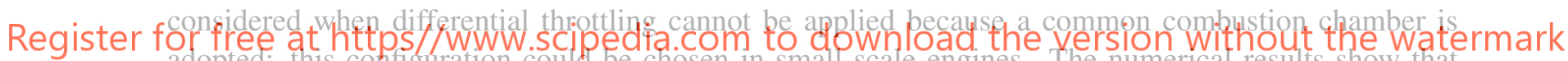
adopted: this configuration could be chosen in small scale engines. The numerical results show that

shock vectoring is quite effective since it is possible to generate a significant lateral thrust component by injecting a few percentage points of the primary mass flow. The simulations show that the location of the secondary injection is a key parameter for two reasons. The first reason is that the injection induces a compression upstream to the injection slot and an expansion downstream to the injection slot: if the slot is located far from the end of the plug then the two effects tend to cancel each other and this reduces the magnitude of the lateral thrust component. This numerical result is in line with the experimental findings of [5] who underlined the importance of placing the injection slot close to the end of the plug.

\section{REFERENCES}

[1] C. Bach, S. Schöngarth, B. Bust, M. Propst, J. Sieder-Katzmann, and M. Tajmar, How to steer an aerospike, in 69th International Astronautical Congress (IAC), Bremen, Germany, 2018.

[2] E. Besnard, H. H. Chen, T. Mueller, and J. Garvey, Design, manufacturing and test 


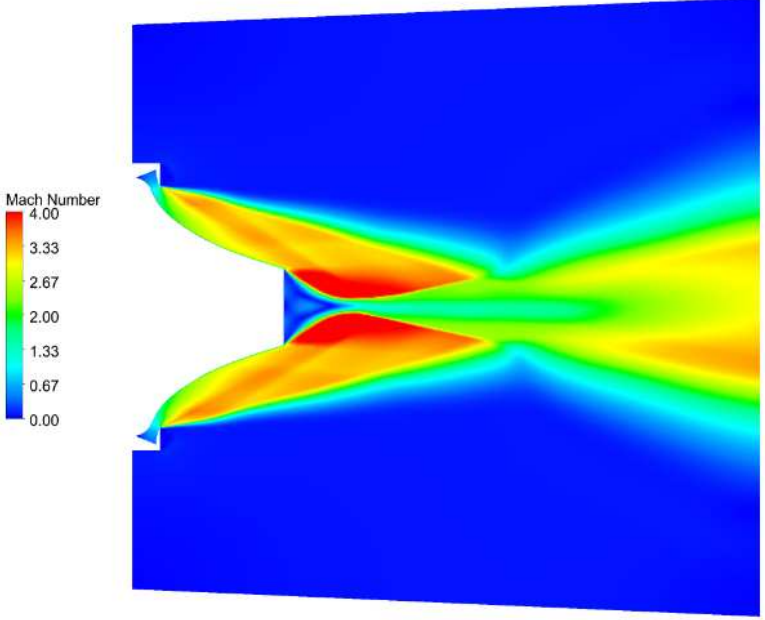

(a)
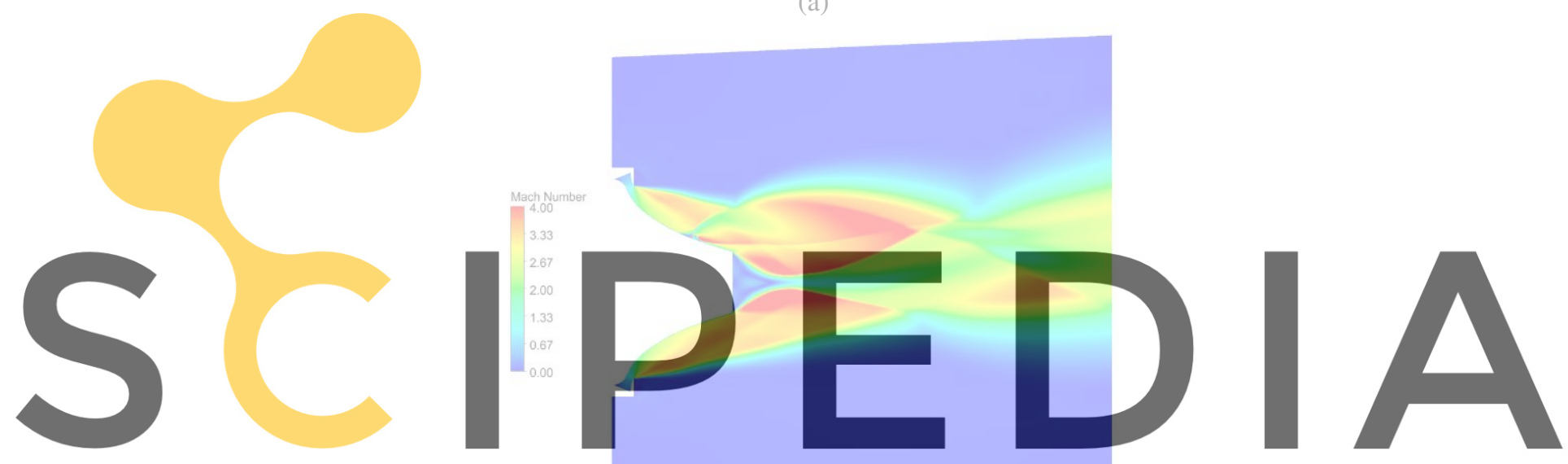

Register for free at https//www.scipedia.com to download the version without the watermark (b)

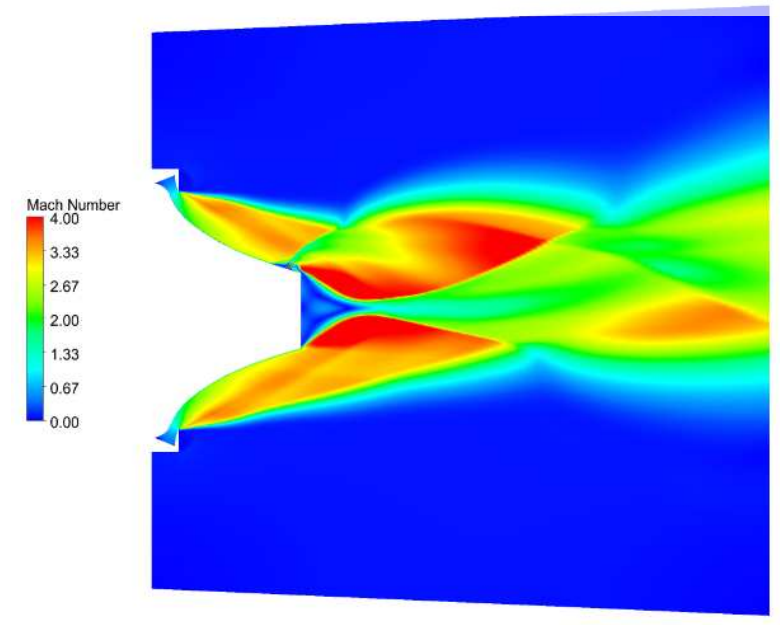

(c)

Figure 3: Mach field for LP40 with differential throttling with $\dot{m}_{2} / \dot{m}_{1}=0.1$ (a), SVC at $x / L=0.6$ with $\dot{m}_{i} / m_{1}$ (b) and SVC at $x / L=0.9$ with $\dot{m}_{i} / m_{1}=0.1$ (c). 


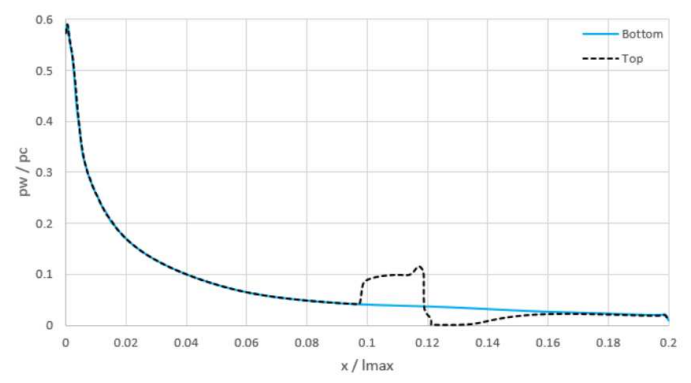

(a)

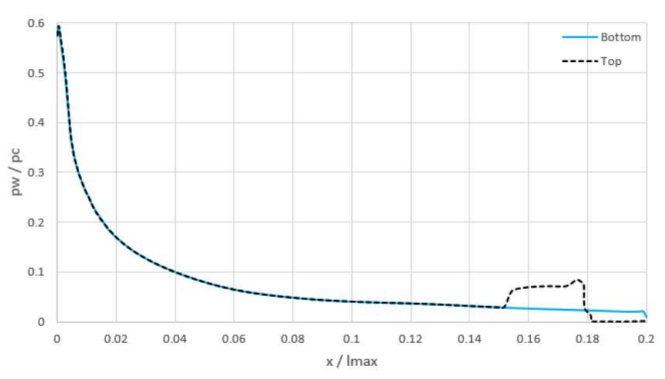

(b)

Figure 4: Wall pressure distribution on LP40 with $\dot{m}_{i} / m_{1}=0.1$ with injection slot at $x / L=0.6$ (a) and $x / L=0.9$ (b).
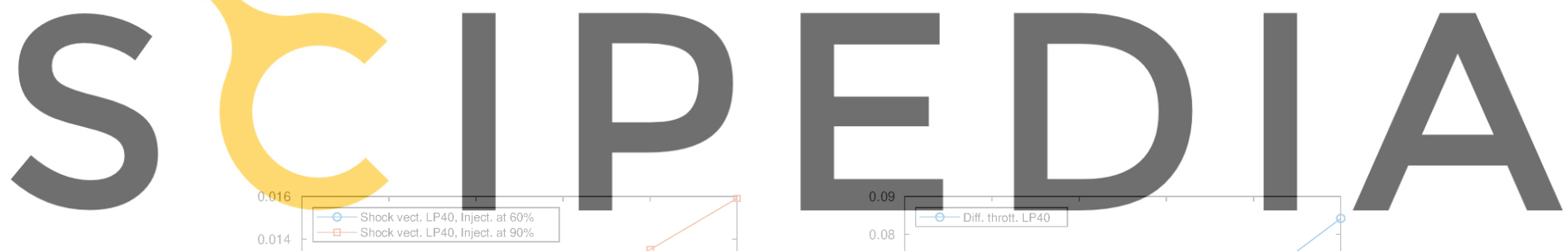

Register for free at https//www.scipedia.com to downlload the version without the watermark

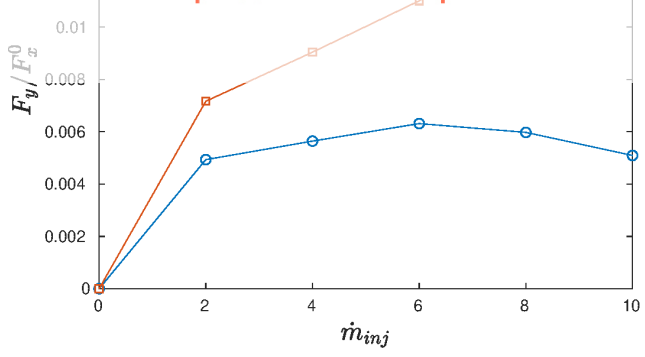

(a)

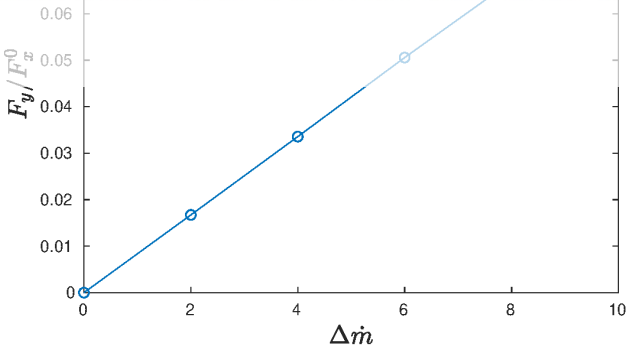

(b)

Figure 5: Side force component obtained by shock vector control (a) and differential throttling (b) on LP40 geometry. 
of a plug nozzle rocket engine, in 38th AIAA/ASME/SAE/ASEE Joint Propulsion Conference \& Exhibit, 2002, p. 4038.

[3] M. G. DAgostino, Y. C. Lee, T.-S. WAng, And J. Turner, X-33 xrs-2200 linear aerospike engine sea level plume radiation, 2001. Technical report.

[4] G. Dumnov, G. NikUlin, And N. Ponomaryov, Investigation of advanced nozzles for rocket engines, Space Rocket Engines and Power Plants, 4 (1993), pp. 10-12.

[5] S. Eilers, W. MATthew, AND S. WhitMore, Analytical and experimental evaluation of aerodynamic thrust vectoring on an aerospike nozzle, in 46th AIAA/ASME/SAE/ASEE Joint Propulsion Conference \& Exhibit, 2010, p. 6964.

[6] S. D. Eilers, M. D. Wilson, S. A. Whitmore, And Z. W. Peterson, Side-force amplification on an aerodynamically thrust-vectored aerospike nozzle, Journal of Propulsion and Power, 28 (2012), pp. 811-819.

[7] N. ERNI, Closed-loop attitude control using fluid dynamic vectoring on an aerospike nozzle, 2011. Thesis, Utah State University.

[8] M. Ferlauto, A. Ferrero, And R. Marsilio, Shock vector control technique for aerospike nozzles, in AIAA Scitech 2020 Forum, 2020, p. 2246.

[9] M. Ferlauto And R. Marsilio, Numerical investigation of the dynamic characteristics of a dual-throat-nozzle for fluidic thrust-vectoring, AIAA Journal, 55 (2017), pp. 86-98.

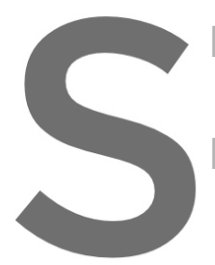

[10] G. Hagemann, Journal of Propulsion

11] Z. HAO, H. TIAN of throttleable hybria
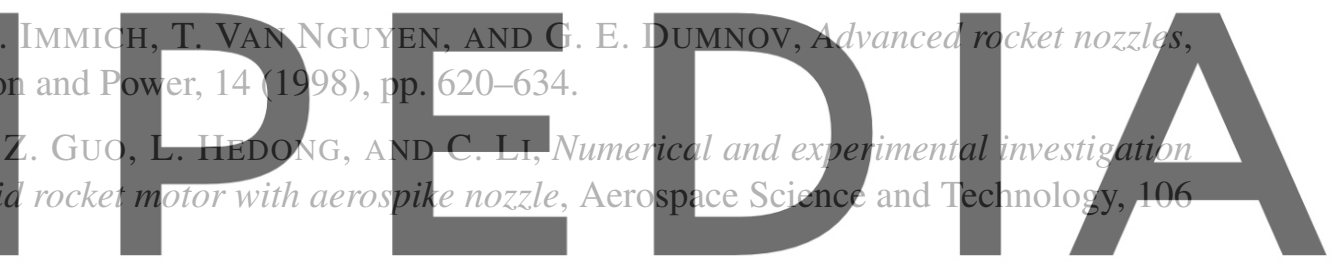

[12] H. Immich, M. CAPoricCi, H. Immich, ANd M. CAPoricci, Status of the festip rocket propul-

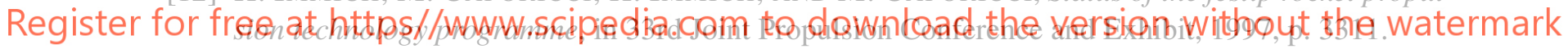

[13] D. MAnski, Clustered plug nozzles for future european reusable rocket launchers, German Aerospace Research Establishment, (1981), pp. 643-81.

[14] R. Marsilio, M. Ferlauto, And M. H. Hamedi-Estakhrsar, Numerical simulation of a vectored axisymmetric nozzle, in AIP Conference Proceedings, vol. 2293, AIP Publishing LLC, 2020, p. 200018.

[15] M. Propst, J. Sieder, C. BACH, AND M. TAJMAR, Numerical analysis on an aerodynamically thrust-vectored aerospike nozzle, in Proceedings of the 63rd German Aerospace Congress (DGLR), Augsburg, 2014.

[16] P. Reijasse AND B. GoRbel, Experimental analysis of the supersonic flow confluence past a jet-on axisymmetric afterbody, in 14th Applied Aerodynamics Conference, 1996, p. 2449.

[17] P. L. RoE, Approximate riemann solvers, parameter vectors, and difference schemes, Journal of computational physics, 43 (1981), pp. 357-372.

[18] J. Siedera, C. BACh, M. Propstc, AND M. TAJMAR, Evaluation of the performance potential 
of aerodynamically thrust vectored aerospike nozzles, in 67th International Astronautical Congress (IAC), Guadalajara, Mexico, 26-30 September 2016, 2016.

[19] R. SILVER, Advanced aerodynamic spike configurations, tech. rep., Rocketdyne, Canoga Park, United States, 1966.

[20] P. Spalart And S. Allmaras, A one-equation turbulence model for aerodynamic flows, Recherche Aerospatiale, 1 (1994), pp. 5-21.

[21] H. Takahashi, S. Tomioka, N. Sakuranaka, T. Tomita, K. Kuwamori, and G. MaSUYA, Experimental study on the aerodynamic performance of clustered linear aerospike nozzles, in 18th AIAA/3AF International Space Planes and Hypersonic Systems and Technologies Conference, 2012, p. 5933.

[22] T. TOMita, H. TAMURA, AND M. TAKAHASHI, An experimental evaluation of plug nozzle flow field, in 32nd Joint Propulsion Conference and Exhibit, 1996, p. 2632.
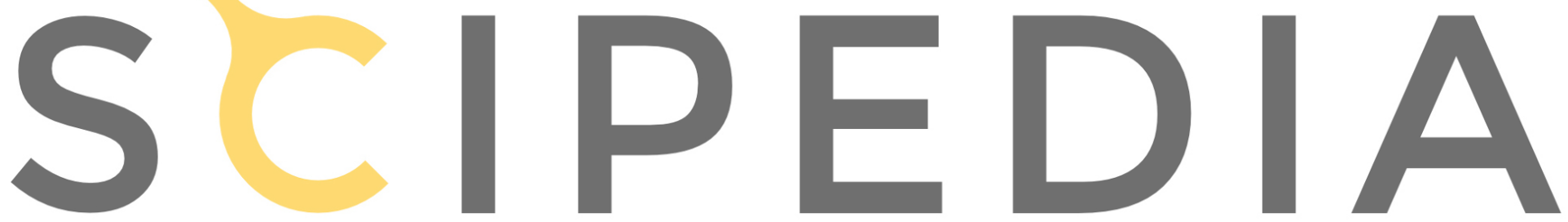

Register for free at https//www.scipedia.com to download the version without the watermark 